\title{
ChemR23 knockout mice display mild obesity but no deficit in adipocyte differentiation
}

\author{
Laurie Rouger ${ }^{1}$, G Raphaël Denis ${ }^{3}$, Souphalone Luangsay ${ }^{1,4}$ and Marc Parmentier ${ }^{1,2}$ \\ ${ }^{1}$ Institut de Recherche Interdisciplinaire en Biologie Humaine et Moléculaire and ${ }^{2}$ WELBIO, Université Libre de \\ Bruxelles, Campus Erasme, Route de Lennik 808, B-1070 Brussels, Belgium \\ ${ }^{3}$ Université Paris Diderot, Sorbonne Paris-Cité BFA, CNRS EAC 4413, F-75205 Paris, France \\ ${ }^{4}$ Euroscreen SA, Brussels, Belgium
}

Correspondence should be addressed to M Parmentier Email

mparment@ulb.ac.be

\begin{abstract}
Chemerin was initially described as a chemoattractant factor for leukocyte populations. More recently, the protein has also been reported to be an adipokine, regulating adipocyte differentiation in vitro via its receptor ChemR23, and to be correlated with BMI and other parameters of the metabolic syndrome in humans. The aim of this study was to investigate the role of the chemerin/ChemR23 axis in the regulation of metabolism in vivo, using a mouse knockout (KO) model for ChemR23 (Cmklr1) in a C57BL/6 genetic background. Body weight and adipose tissue mass did not differ significantly in young animals, but were significantly higher in ChemR23 KO mice aged above 12 months. Glucose tolerance was unaffected. No significant modifications in the levels of blood lipids were observed and no increase in the levels of inflammatory markers was observed in the adipose tissue of KO mice. A high-fat diet did not exacerbate the obese phenotype in ChemR23 KO mice. No obvious defect in adipocyte differentiation was detected, while a marker of lipogenic activity (GPD1 expression) was found to be elevated. In conclusion, the chemerin/ChemR23 system does not appear to play a major role in adipocyte differentiation in vivo, but it may be involved in adipose tissue homeostasis.
\end{abstract}

\author{
Key Words \\ - G-protein-coupled receptor \\ - adipokines \\ - inflammation
}

Journal of Endocrinology (2013) 219, 279-289

\section{Introduction}

Obesity and associated disorders, such as type 2 diabetes, metabolic syndrome, and cardiovascular diseases, constitute a rapidly growing health problem in Western countries. According to the World Health Organization, worldwide obesity has doubled since 1980 (Pi-Sunyer 2002, Karlsson \& Beck 2010). In this context, a better understanding of adipose tissue biology could contribute to the control of energy metabolism and obesity, at least in the most extreme situations. White adipose tissue (WAT) contributes to endocrine signaling by the secretion of a set of factors, collectively named adipokines, expressed and secreted by adipocytes and delivered locally and into the systemic circulation. Adipokines have various functions in the control of metabolism, insulin responses, and inflammation (Galic et al. 2010). Some adipokines also influence adipose tissue development and inflammatory cell recruitment. Leptin and adiponectin are the most studied members of the adipokine family (Maffei et al. 1995, Hu et al. 1996), but several new members have been discovered recently (MacDougald \& Burant 2007, Wozniak et al. 2009, Ouchi et al. 2011), and since 2007, chemerin has been included in this family of extracellular

Published by Bioscientifica Ltd. 
mediators (Bozaoglu et al. 2007, Goralski et al. 2007, Ernst $\&$ Sinal 2010).

Chemerin is a chemoattractant protein synthesized and secreted as an inactive precursor, prochemerin, which is activated by various serine proteases, including cathepsin $G$, neutrophil elastase, and proteases of the coagulation cascade (Wittamer et al. 2003). Chemerin acts via its G-protein-coupled receptor ChemR23, expressed in dendritic cells and macrophages, and displays antiinflammatory properties (Luangsay et al. 2009, Bondue et al. 2011). Strong expression of chemerin and ChemR23 in human and murine adipose tissue (Bozaoglu et al. 2007, Goralski et al. 2007, Mussig et al. 2009) indicates that chemerin might also act as an adipokine. In humans, blood chemerin levels have been described to be correlated with BMI, circulating triglyceride levels, blood pressure, and various other parameters of the metabolic syndrome (Goralski et al. 2007, Bozaoglu et al. 2009, Mussig et al. 2009, Sell et al. 2010). In mice, the expression of chemerin in adipocytes is upregulated under a high-fat diet (HFD; Roh et al. 2007, Ernst et al. 2010) and is increased in obese/diabetic $d b / d b$ mice (Ernst et al. 2010, Parlee et al. 2010) and in diabetic Psammomys obesus (Goralski et al. 2007). The administration of recombinant chemerin has also been reported to reduce glucose uptake, increasing glucose intolerance in $d b / d b$ mice and diet-induced obesity models (Ernst et al. 2010). Recently, Ernst et al. (2012) have reported that regardless of diet, ChemR23 (Cmklr1) knockout (KO) mice display lower food intake, body mass, and body fat percentage, with decreased hepatic and adipose tissue inflammation and relative glucose intolerance. Many in vitro studies have reported the effects of the chemerin/ChemR23 system on adipocytes. Chemerin has been proposed to control the differentiation of 3T3-L1 preadipocytes via ChemR23 (Bozaoglu et al. 2007, Goralski et al. 2007, Roh et al. 2007, Muruganandan et al. 2010), to promote lipolysis (Roh et al. 2007), and to potentiate insulin-stimulated glucose uptake in adipocytes (Takahashi et al. 2008). Taken together, these previous studies indicate a role for the chemerin/ ChemR23 system in adipose tissue function and metabolism. However, many aspects remain controversial, as results are often conflicting according to studies, even when using very similar models.

The aim of this study was to investigate further in vivo whether chemerin and ChemR23 play a role in the regulation of body weight and energy metabolism in mice, by acting on adipocyte differentiation and function, insulin sensitivity, and inflammatory status.

\section{Materials and methods}

\section{Mice}

As has been described previously (Luangsay et al. 2009), ChemR23 KO mice were obtained from Deltagen (San Mateo, CA, USA) and backcrossed for 13 generations into a C57BL/ 6 background. Heterozygous mice (ChemR23 ${ }^{+/-}$) were then interbred to generate ChemR23 KO mice $\left(\right.$ ChemR23 $\left.3^{-/-}\right)$and their WT controls $\left(\right.$ChemR $\left.23^{+/+}\right)$. The mice were kept in a specific pathogen-free (SPF) environment under a $12 \mathrm{~h}$ light: $12 \mathrm{~h}$ darkness cycle with unlimited access to food and water. The mice were fed standard laboratory diet with $3.5 \%$ fat (Carfil, OudTurnhout, Belgium). Food intake was recorded in 4-month-old mice as the difference between preweighed food and food remaining $48 \mathrm{~h}$ later. Blood and tissue samples were collected under anesthesia at different ages from three different groups of mice to evaluate the effects of invalidation during life: young (12 weeks), middle-aged (8 months), and old (14 months). All the procedures involving animals were reviewed and approved by the local ethics committee (Commission d'Ethique du Bien-Etre Animal, Université Libre de Bruxelles).

\section{Adipose tissue analysis}

Inguinal fat pads were collected and weighed, and the right pads were processed for histology or stored at $-80^{\circ} \mathrm{C}$ for RNA extraction. Histological samples were fixed in $4 \%$ paraformaldehyde (PFA), dehydrated, and embedded in paraffin. Sections $(7 \mu \mathrm{m})$ were stained with hematoxylin and eosin and assessed by light microscopy. The section area of adipocytes was measured using the ImageJ Software (National Institute of Health, Bethesda, MD, USA). Total DNA was extracted from $25 \mathrm{mg}$ of frozen samples of adipose tissue using the DNeasy Kit (Qiagen, Hilden, Germany) and eluted using $200 \mu \mathrm{l}$ of buffer; the result of the assays was analyzed as an estimate of the total number of cells (adipocytes, immature cells, immune cells, etc.) for the same weight of fat. Total RNA was extracted from frozen tissue using the RNeasy Kit (Qiagen). First-strand cDNA was synthesized using Superscript III reverse transcriptase and random hexamers as primers following the manufacturer's instructions (Invitrogen). Samples of cDNA were diluted 1:25 in nuclease-free water, and real-time quantitative PCR was carried out in a $20 \mu \mathrm{l}$ volume, containing $8 \mu \mathrm{l}$ diluted cDNA template, $1.25 \mu \mathrm{M}$ forward and reverse primers, and PCR SYBR Green I Mix (Qiagen). PCR conditions were $95^{\circ} \mathrm{C}$ for $15 \mathrm{~min}$, followed by 40 cycles of $94^{\circ} \mathrm{C}$ for $15 \mathrm{~s}, 58^{\circ} \mathrm{C}$ for

Published by Bioscientifica Ltd 
$20 \mathrm{~s}$, and $72{ }^{\circ} \mathrm{C}$ for $20 \mathrm{~s}$ in a 7500 Fast Real Time PCR system (Applied Biosystems). After amplification, melting curve analysis was carried out, and relative expression values were calculated using the $\Delta \Delta C$ t method and the Canx and Ywhaz genes as controls. The sequences of primers were as follows: Tnf $\alpha$ (Tnf) forward, 5'-CAGACCCTCACACTCAGATCA-3'; Tnf $\alpha$ reverse, 5'-CACTTGGTGGTTTGCTACGA-3'; Il1 $\beta$ (Illb) forward, 5'-CAACCAACAAGTGATATTCTCCATG-3'; Ill $\beta$ reverse, 5'-GATCCACACTCTCCAGCTGCA-3'; Il6 forward, 5'-GAGGATACCACTCCCAACAGACC-3'; $I l 6$ reverse, 5'-AAGTGCATCATCGTTGTTCATACA-3'; Ppary (Pparg) forward, 5'-TCCGAAGAACCATCCGATT-3'; Ppar $\gamma$ reverse, $5^{\prime}$-TTTGTGGATCCGGCAGTTAAG- ${ }^{\prime}$; Dlk1 forward, 5'-CGTGATCAATGGTTCTCCCT-3'; Dlk1 reverse, 5'-AGGGGTACAGCTGTTGGTTG-3'; lipin 1 forward, 5'-CCATTCACAGCGAGTCTTCA- $3^{\prime}$; lipin 1 reverse, $5^{\prime}$-TGGAAGGGGAATCTGTCTTG-3'; Gpd1 forward, 5'-ATGCTCGCCACAGAATCCACAC-3'; Gpd1 reverse, 5'-AACCGGCAGCCCTTGACTTG-3'; Lpl forward, 5'-GGGCTCTGCCTGAGTTGTAG-3'; Lpl reverse, 5'-CCATCCTCAGTCCAAGAAAA-3'; chemerin (Rarres2) forward, 5'-CCAACTGCCCCAAGAAGGA-3'; chemerin (Rarres2) reverse, 5'-AATGCAGGCCAGGCATTTC-3'; ChemR23 forward, 5'-GTTCAACATCTTTTTGCCGAT-3'; ChemR23 reverse, 5'-TCCCGAACACCCAGTGGTA-3'; Gpr1 forward, 5'-GTGGTCATTGCCTTCTTGGT-3'; Gpr1 reverse, 5'-GGCTCTTGGTTTCAGCACTC-3'; Canx forward, 5'-TTGCTGACTCCTTTGACAGAGG3'; Canx reverse, 5'-CCACTTTCCATCATATTTGGCA-3'; Ywhaz forward, 5'-TGCAACGATGTACTGTCTCTTTTG-3'; and Ywhaz reverse, 5'-CGGTAGTAGTCACCCTTCATTTTCA-3'.

\section{In vitro studies}

Freshly collected adipose tissue samples were digested using collagenase I (Sigma-Aldrich) at $37^{\circ} \mathrm{C}$ for 40-60 $\mathrm{min}$, with intermittent pipetting. The suspension was added to $30 \mathrm{ml}$ DMEM/F12 and passed through a $100 \mu \mathrm{m}$ filter. The filtrate was centrifuged, and the pellet was resuspended in DMEM/F12. Red blood cells were lysed using RBC lysis buffer, and after lavage, the final pellet was resuspended in $1 \mathrm{ml}$ of $3 \%$ DMEM/calf serum and 300000 cells were seeded per each well in a 12-well plate and incubated at $37^{\circ} \mathrm{C}$ in a $5 \% \mathrm{CO}_{2}$ incubator. After 1 week, adipocyte differentiation was induced by the addition of $5 \mu \mathrm{g} / \mathrm{ml}$ insulin, $50 \mathrm{mM}$ indomethacin, $1 \mu \mathrm{g} / \mathrm{ml}$ dexamethasone, $100 \mathrm{mM}$ 3-isobutyl-1-methylxanthine (IBMX) in 3\% DMEM/calf serum for 1 week. The cells were fixed using 4\% PFA, and lipid droplets were stained using Oil Red O to quantify differentiation. Microscopic images were taken using an inverted light microscope, and the number of differentiated cells was determined using the ImageJ Software and compared with the total number of cells in each well.

\section{Cytokine assays}

The mice that were fasted for $16 \mathrm{~h}$ were anesthetized using thiopental $(10 \mathrm{mg} / \mathrm{kg})$, and blood was collected from the portal vein. Serum was immediately separated and frozen at $-20{ }^{\circ} \mathrm{C}$ until use. Triglyceride and cholesterol levels were determined on an automated clinical chemistry analyzer in the Erasme Hospital. Insulin (Mercodia, Uppsala, Sweden), and leptin, adiponectin, and chemerin (R\&D Systems, Abington, UK) levels were determined by ELISA, according to the manufacturer's instructions. Serum samples collected from the HFD-fed mice were assayed for TNF $\alpha$ and IL6 levels using a cytometric bead array-based immunoassay (CBA Flex Sets, BD Biosciences Europe, Erembodegem, Belgium), a dual-laser flow cytometer FACSCalibur (BD Biosciences Europe), and the FCAP Array Software (BD Biosciences Europe), following the manufacturer's instructions.

\section{Indirect calorimetry}

The mice were analyzed for whole energy expenditure, oxygen consumption, and carbon dioxide production $\left(V \mathrm{O}_{2}\right.$ and $V \mathrm{CO}_{2}$, where $V$ is volume), respiratory exchange rate $\left(\mathrm{RER}=\mathrm{VCO}_{2} / \mathrm{VO}_{2}\right)$, and spontaneous activities using calorimetric cages with bedding, food, and water (Labmaster, TSE Systems GmbH, Bad Homburg, Germany). The mice were allowed to acclimatize in individual cages for $48 \mathrm{~h}$ before the experiment. Data were recorded every 40 min during the entire experiment. The ratio of gas concentrations was determined using an indirect opencircuit calorimeter (Arch et al. 2006), which can monitor $\mathrm{O}_{2}$ and $\mathrm{CO}_{2}$ concentrations. The flow was calibrated with $\mathrm{O}_{2}$ and $\mathrm{CO}_{2}$ mixtures of known concentrations before the start of the experiment. Total energy expenditure was calculated according to the Weir equation (Weir 1949) normalized to lean body mass. The mice had free access to food and water ad libitum. Cages were equipped with infrared light beams, allowing the measurement of the locomotor activity of mice throughout the experiment.

\section{High-fat diet}

For 20 weeks (starting at the age of 5 weeks), the mice were fed a HFD in which $46 \%$ of the calories were derived from

Published by Bioscientifica Ltd 
lipids (Purified diet 235HF, Safe, Augy, France). Control mice were fed a standard pellet diet in which $5 \%$ of the calories were provided as fat (Diet 210, Safe). The mice were weighed weekly until the 19th week, and blood was collected under anesthesia after 20 weeks. After coagulation, the serum was separated and frozen at $-20^{\circ} \mathrm{C}$ until use. Inguinal fat pads were frozen in liquid nitrogen and stored at $-80{ }^{\circ} \mathrm{C}$ for subsequent quantitative RT-PCR (qRT-PCR) analysis.

\section{Insulin and glucose tolerance tests}

Insulin and glucose tolerance tests were carried out on 8 -week-old mice fasted for respectively 4 and $16 \mathrm{~h}$. After an i.p. injection of recombinant human insulin (Actrapid, Novo Nordisk, Copenhagen, Denmark; $0.5 \mathrm{mU} / \mathrm{g}$ body weight) or glucose ( $2 \mathrm{mg} / \mathrm{g}$ body weight), blood was collected from the tail vein and glucose levels were measured using a glucometer (One Touch Ultra, Lifescan, Beerse, Belgium) before and 30, 60, and $120 \mathrm{~min}$ after glucose injection or 30,45, and $60 \mathrm{~min}$ after insulin injection.

\section{Statistical analyses}

Data are expressed as means \pm s.E.M. Unpaired two-tailed Student's $t$-test, two-way ANOVA, or Mann-Whitney $U$ tests were used to determine statistical significance depending on the normality of the data. All the statistical analyses were carried out using Statview (SAS Institute, Cary, NC, USA, v 5.0.1) considering a $P$ value of $<0.05$ as significant.

\section{Results}

\section{Progressive and moderate obesity develops in ChemR23 KO mice}

To determine the role of the chemerin/ChemR23 system in metabolism in mice, we first measured the body weight of ChemR23 KO mice and their littermate controls fed a regular (low-fat) chow at three different endpoints in three different groups of male mice: young (12 weeks), middle age ( 8 months), and old (14 months), to determine any difference depending on age. As shown in Fig. 1A, no significant difference was observed in 12-week-old mice, but the weight of male KO mice increased faster than that of control mice, and by 14 months, their weight was, on average, $10 \%$ higher than that of control mice $(31.9 \pm 0.8$ vs $28.8 \pm 0.7 \mathrm{~g}$, respectively, $P=0.01$ ). Interestingly, this moderately obese phenotype was absent in female mice
(Supplementary Figure 1, see section on supplementary data given at the end of this article). Food intake was recorded in 4-month-old male mice, but no significant difference could be observed (Fig. 1B). Inguinal fat pads were collected from 12-week-, 8-week-, and 14-month-old male mice, as a marker of WAT mass. The weight of the fat pads was higher in ChemR23 KO mice at 8 months and was $60 \%$ higher at 14 months (Fig. 1C, $P=0.0079$ ). Histological examination of fat tissue (Fig. 1D) revealed adipocytes of increased diameter in 14-month-old KO mice (Fig. 1E, $P=0.02$ ), while the estimation of the number of cells in WAT by the quantification of DNA content revealed no apparent change in this parameter (Fig. 1F).

Blood lipid, glucose, and insulin levels were measured in 12-week-old (before the onset of mild obesity in ChemR23 KO mice) and 14-month-old, regular chow-fed male mice that were overweight. Cholesterol levels were found to change with age, but were not affected by the genotype (Table 1). Similarly, triglyceride values, glucose levels, and insulin levels were not significantly different between the ChemR23 KO and WT mice.

Because activity-related energy expenditure is a main contributor to energy balance, we examined locomotor activity and energy expenditure by indirect calorimetry in 4-month-old male mice (before the onset of the mild obesity phenotype in ChemR23 KO mice). No difference was detected in ambulatory activity in the dark and light phases (Supplementary Figure 2A, see section on supplementary data given at the end of this article), carbon dioxide production (Supplementary Figure 2B), oxygen consumption (Supplementary Figure 2C), or respiratory quotient (Supplementary Figure 2D), indicating that the lack of ChemR23 does not modify the type of substrate used for energy consumption.

The circulating levels of chemerin and two other major adipokines (leptin and adiponectin) were measured in 14-month-old WT and KO male mice. As shown in Fig. 2, serum adiponectin levels were similar in both the genotypes (Fig. 2A), and serum leptin levels were increased in ChemR23 KO mice without reaching significance (Fig. 2B). Interestingly, the total chemerin levels were decreased by $34 \%$ in ChemR $23 \mathrm{KO}$ mice (Fig. 2C, 316 \pm 25 vs $210 \pm 23 \mathrm{ng} / \mathrm{ml}$ for control mice, $P=0.029$ ), despite the higher body weight of these mice.

\section{Influence of HFD}

To determine whether ChemR23 KO mice would display more overt obesity on being fed hypercaloric diets, mice (5 weeks old) were fed a HFD (46\% lipid-derived calories)

Published by Bioscientifica Ltd. 
A
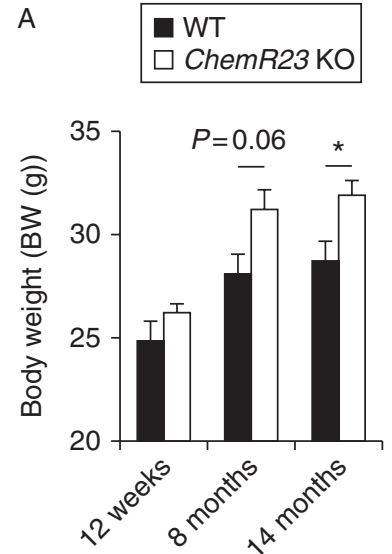

$\mathrm{D}$

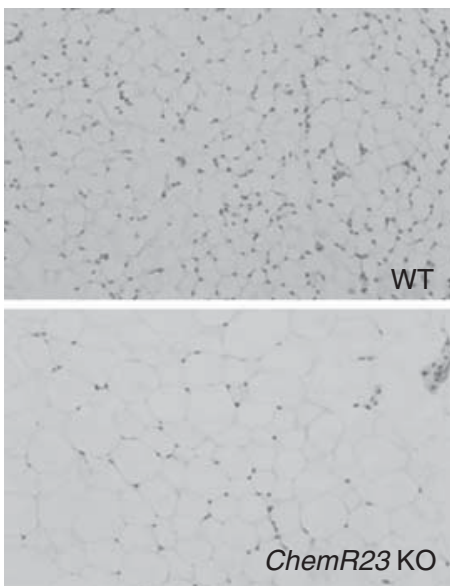

B

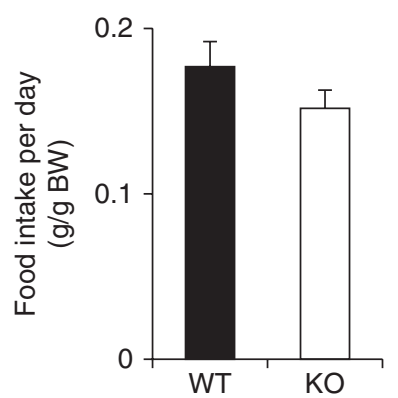

$\mathrm{E}$

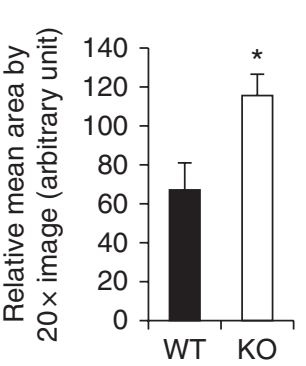

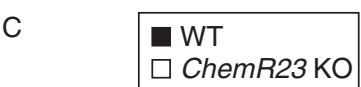

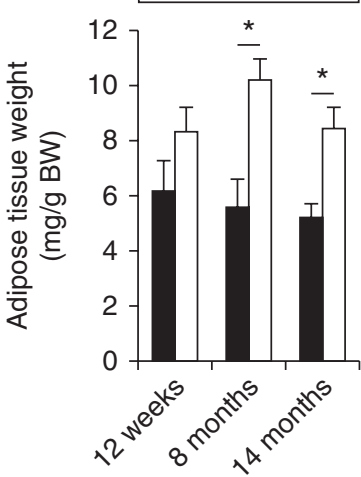

$\mathrm{F}$

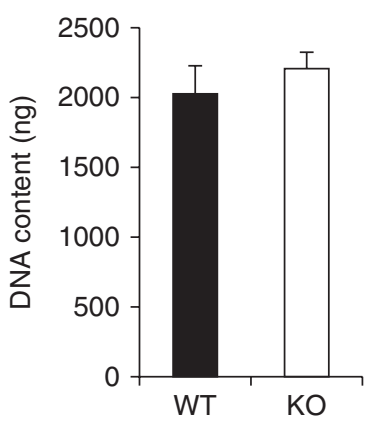

\section{Figure 1}

Body weight and adipose tissue mass of ChemR23 KO mice. (A) Male mice were weighed at 12 weeks ( $n=6$ per group), 8 months ( $n=4$ per group), and 14 months (WT, $n=8 ; \mathrm{KO}, n=15$ ). (B) Daily food intake was recorded in 4-month-old WT and KO male mice ( $n=6$ per group). (C) Inguinal adipose tissue samples were collected from 12-week-old ( $n=6$ per group), 8-weekold ( $n=4$ per group), and 14-month-old (WT, $n=8 ; \mathrm{KO}, n=15$ ) male mice and weighed. (D) Histology of inguinal adipose tissue samples collected from 14-month-old WT and ChemR23 KO mice was studied after

for 20 weeks. As shown in Fig. 3A, the HFD-fed WT and ChemR23 KO mice exhibited a higher increase in body weight compared with their respective controls fed a standard diet after 19 weeks. However, weight increment was not higher in ChemR23 KO mice. After 19 weeks, there hematoxylin and eosin staining of paraffin sections (original magnification, $20 \times)$. (E) Mean section area of adipocytes was determined in the sections collected from WT and ChemR23 KO mice (WT, $n=4 ; \mathrm{KO}, n=6$ ). (F) DNA content (ng) of the adipose tissue of WT and ChemR23 KO mice was determined ( $n=8$ per group). Black columns, WT mice; white columns, ChemR23 KO mice. The unpaired two-tailed Student's $t$-test was used, $* P<0.05$.

was no difference in the body weight of the standard dietfed WT mice and HFD-fed mice. An increase in the levels of cholesterol (Fig. 3B), mainly associated with LDLs was observed, with no difference being observed with regard to genotype.

Table 1 Metabolic parameters of 12-week-old and 14-month-old WT and ChemR23 KO mice. $n=4-8$ per group

\begin{tabular}{|c|c|c|c|c|}
\hline & WT mice (12 weeks) & $\begin{array}{c}\text { ChemR23 } \\
\text { KO mice (12 weeks) }\end{array}$ & WT mice (14 months) & $\begin{array}{c}\text { ChemR23 } \\
\text { KO mice (14 months) }\end{array}$ \\
\hline Cholesterol (mg/dl) & $96.0 \pm 2.6$ & $102 \pm 6.7$ & $64.7 \pm 5.2$ & $70.2 \pm 6.2$ \\
\hline Triglycerides (mg/dl) & $25.7 \pm 13.7$ & $38.0 \pm 11.7$ & $34.9 \pm 3.9$ & $26.4 \pm 7.5$ \\
\hline Glucose (mg/dl) & $120 \pm 5$ & $93 \pm 2$ & $93 \pm 6$ & $91 \pm 3$ \\
\hline Insulin $(\mu \mathrm{g} / \mathrm{l})$ & $0.53 \pm 0.03$ & $0.66 \pm 0.06$ & $0.65 \pm 0.07$ & $0.51 \pm 0.02$ \\
\hline
\end{tabular}



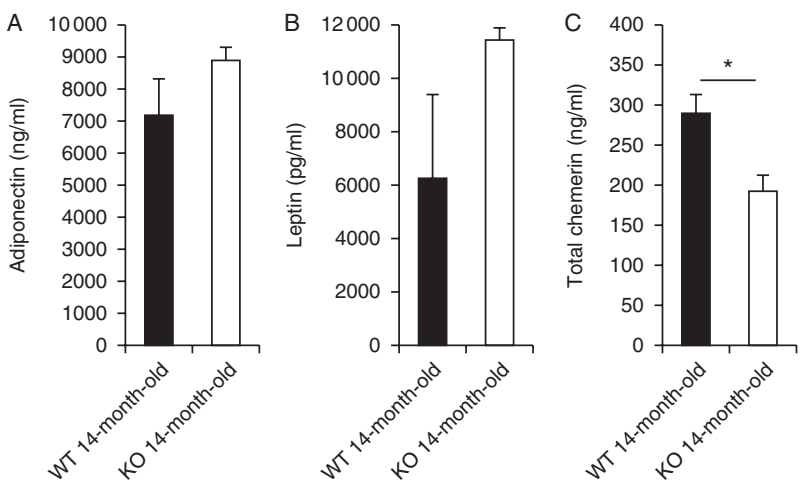

Figure 2

Blood adipokine levels. Adiponectin (A), leptin (B), and total chemerin (C) levels were measured by ELISA of the serum of 14-month-old WT (black columns, $n=8$ ) and KO (white column, $n=15$ ) mice. The nonparametric Mann-Whitney $U$ test was used, ${ }^{*} P<0.05$.
Although blood chemerin levels were not affected by HFD (Fig. 3C), chemerin transcript levels in adipose tissue were significantly increased in the HFD-fed mice independent of genotype (Fig. 3D), in agreement with the findings reported by Roh et al. (2007), and this diet led to an increase in ChemR23 transcript levels in the adipose tissue of WT mice (Fig. 3E); data for ChemR23 $\mathrm{KO}$ mice are not reported because no transcripts were detected. The inflammatory status of the HFD-fed mice was evaluated after 20 weeks, but neither the circulating levels of TNF $\alpha$ and IL6 (Fig. 3F) nor the relative expression of TNF $\alpha$ and IL1 (Fig. 3G) in adipose tissue was altered. Therefore, it appears that under the conditions of our experiment, ChemR23 deficiency and HFD led to an increased body weight gain, but these effects did not appear to be additive.
B

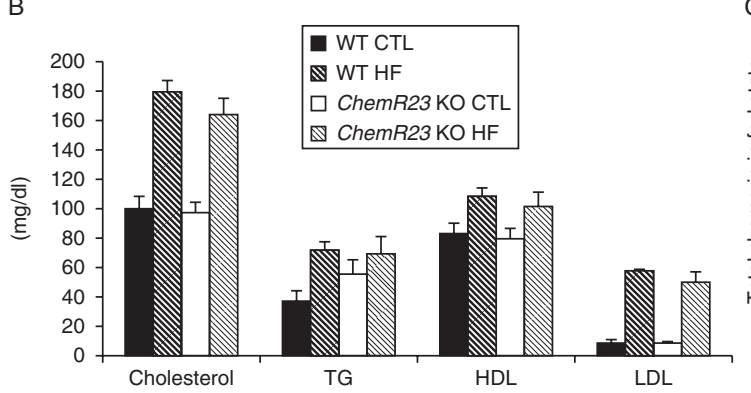

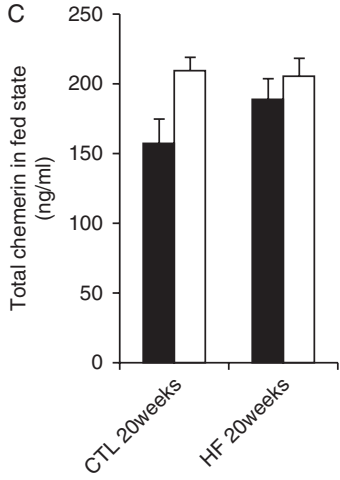
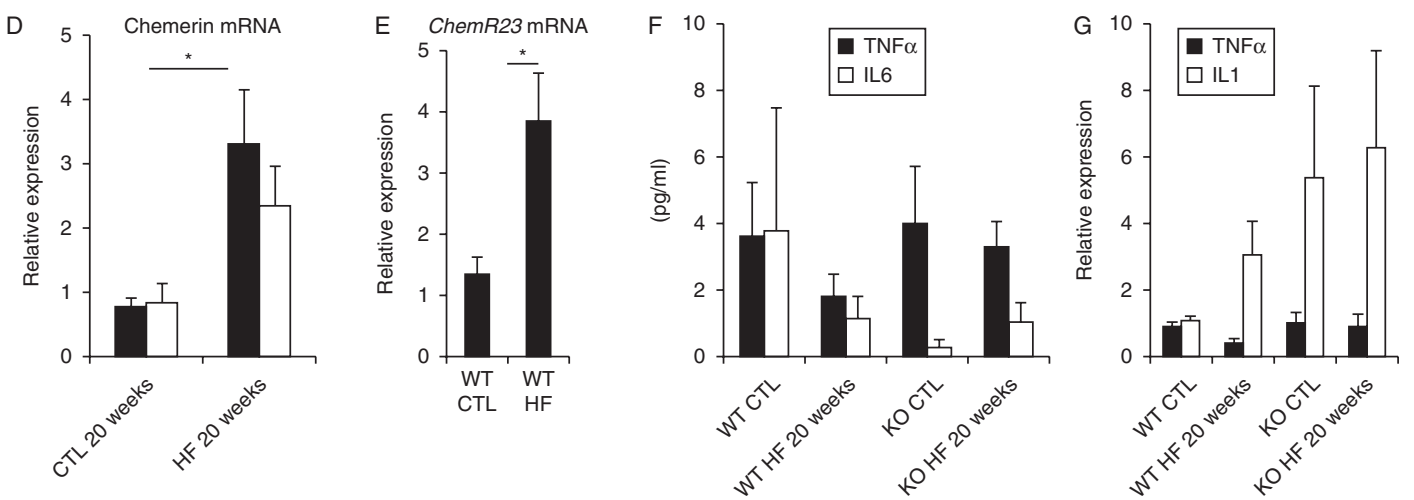

Figure 3

Effects of high-fat diet (HFD) on the body weight, blood lipids, chemerin/ChemR23 expression, and proinflammatory cytokines in adipose tissue. Body weight (A) was measured once per week in CTL WT (black columns, $n=4$ ), HF WT (black columns, $n=9$ ), CTL ChemR23 KO (white columns, $n=5$ ), and HF ChemR23 KO (white columns, $n=10$ ) mice after 4 and 20 weeks of diet. Blood lipid parameters were analyzed after 20 weeks in (B) CTL WT (black columns, $n=4$ ), HF WT (dashed black columns, $n=9$ ), CTL ChemR23 KO (white columns, $n=5$ ), and HF ChemR23
KO (dashed white columns, $n=10$ ) mice. Two-way ANOVA followed by Fisher's post hoc test was used, $* P<0.05$. Chemerin (C) and TNF $\alpha$ and IL6 (F) levels were measured by ELISA of the serum of WT (black columns) and ChemR23 KO (white columns) mice after 20 weeks of being fed the control (CTL) diet or HFD. Relative transcript levels of chemerin (D), ChemR23 (E), and TNF $\alpha$ and ILI (G) were estimated by qRT-PCR of the adipose tissue of the same groups (WT, $n=5$ per group; KO, $n=7$ per group). The nonparametric Mann-Whitney $U$ test was used, ${ }^{\star} P<0.05$. 


\section{Role of the chemerin/ChemR23 system in glucose metabolism}

To investigate the role played by the chemerin/ChemR23 system in glucose homeostasis, we carried out tolerance tests for glucose and insulin in 8-week-old WT and ChemR23 KO mice. At this age, there is no significant difference in body weight. After i.p. injection of glucose, a strong increase in glucose levels was observed at $30 \mathrm{~min}$, followed by a progressive return to baseline by $120 \mathrm{~min}$ (Fig. 4A and B). No difference in the hyperglycemia curves or in the insulin levels measured 30 and 60 min after glucose injection was observed between WT and ChemR23 KO mice (Fig. 4C). Following insulin injection, glucose levels dropped moderately in WT and ChemR23 KO mice (Fig. 4D and E). All these data call into question the anti-insulin properties of the chemerin/ChemR23 system in vivo.

\section{Adipocyte differentiation and activity markers in ChemR23 KO mice}

The qRT-PCR analysis of WAT markers (Fig. 5A) indicated an increased expression of glycerol phosphate dehydrogenase (GPD1), an enzyme involved in lipogenesis, in 14-month-old ChemR23 KO mice, but no changes in the expression of peroxisome proliferator-activated receptor $\gamma$ (PPAR $\gamma$ ) or lipoprotein lipase, implicated in lipolysis, were detected. No differences in the expression of the preadipocyte marker preadipocyte factor 1 (PREF1, Dlk1) and lipin 1 were observed, suggesting that ChemR23 is not essential for adipocyte maturation in vivo. ChemR23 $\mathrm{KO}$ mice exhibited the same level of expression of GPR1 in adipose tissue. The relative expression of proinflammatory cytokines IL1 $\beta$, IL6, and TNF $\alpha$, and chemokine MCP1 (CCL2) was similar for both the genotypes (Fig. 5B). Moreover, in vitro culture of preadipocytes obtained from WT and ChemR23 KO mice resulted in the same level of differentiation after the addition of insulin, dexamethasone, indomethacin, and IBMX (Fig. 5C and D).

\section{Discussion}

In this study, we investigated the role of ChemR23, a G-protein-coupled receptor expressed in human and murine adipose tissue, in glucose and lipid metabolism in vivo. ChemR23 is the receptor of chemerin, described as a new adipokine. Under the standard environmental conditions and diet used in the present study, male (but not female) ChemR23 KO mice developed a moderate
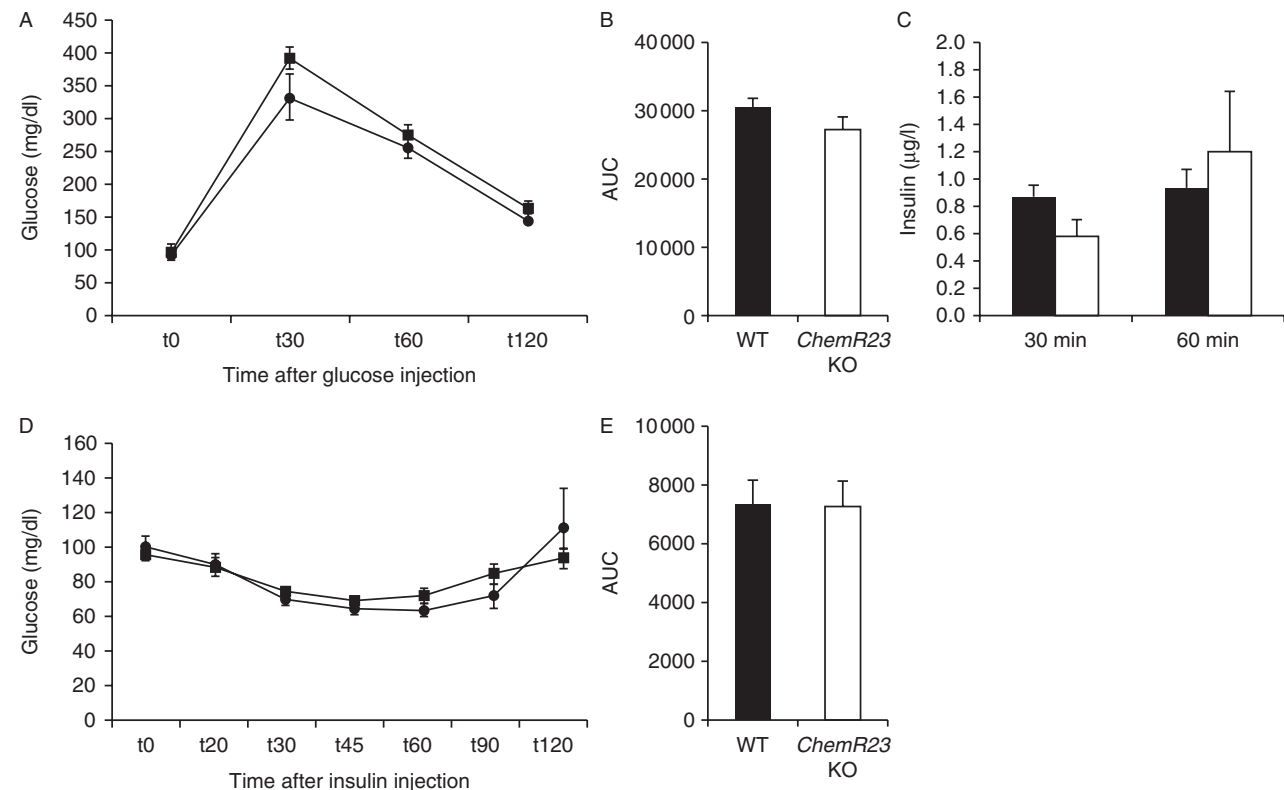

\section{Figure 4}

Results of glucose and insulin tolerance tests. A glucose tolerance test was carried out in 8-week-old mice after a 16-h fast by i.p. injection of glucose ( 2 mg/g body weight). Glucose levels were measured at 30,60, and $120 \mathrm{~min}$ (A, $n=8$ per group) and area under the curve was calculated (B). Blood insulin levels were measured by ELISA at 30 and $60 \mathrm{~min}$ (C, $n=5$ per group). An insulin tolerance test was carried out after a 16-h fast by i.p. injection of human insulin ( $0.5 \mathrm{mU} / \mathrm{g}$ body weight). Glucose levels were measured at 20,30, 45, 60, and $120 \mathrm{~min}$ ( $D, n=12$ per group) and area under the curve was calculated (E). Squares and black columns, WT mice; circles and white columns, ChemR23 KO mice. Nonparametric Mann-Whitney $U$ test was used.

http://joe.endocrinology-journals.org DOI: 10.1530/JOE-13-0106
C 2013 Society for Endocrinology Printed in Great Britain
Published by Bioscientifica Ltd. 


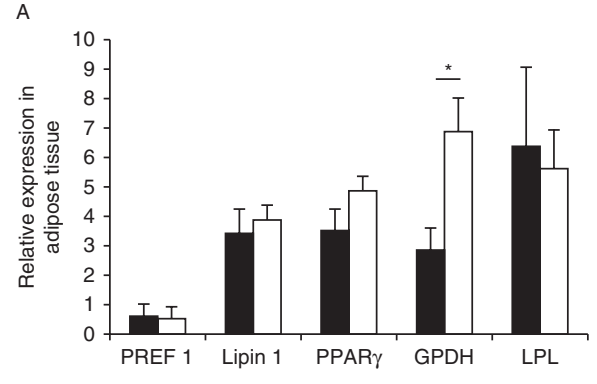

C

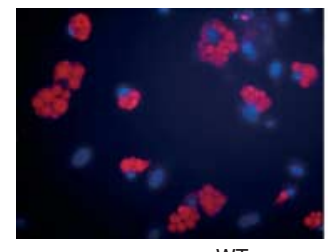

WT

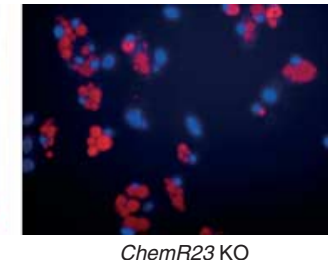

ChemR23 KO
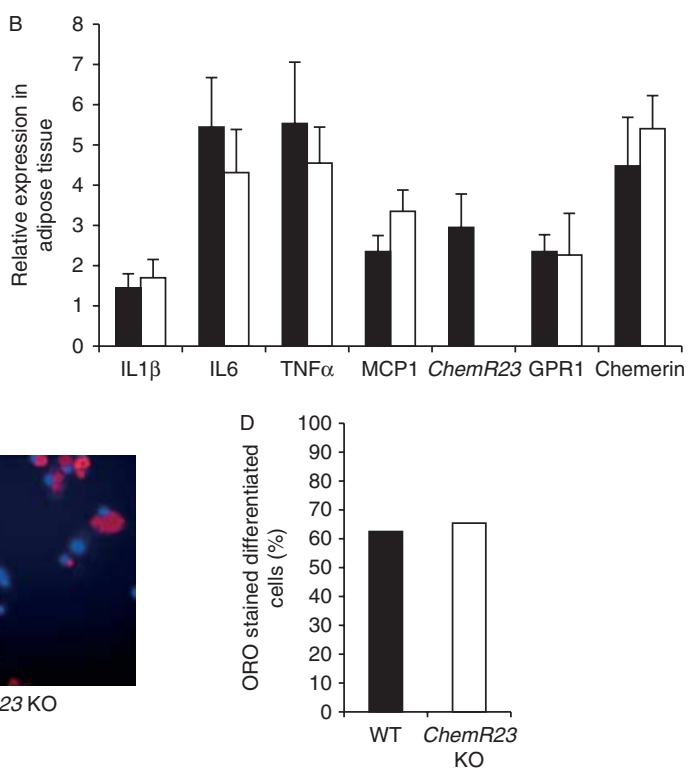

Figure 5

Differentiation and inflammation markers in the adipose tissue of ChemR23 KO and WT mice. The relative expression of the markers of (A) adipocyte differentiation (PREF1, lipin1, and PPAR $\gamma$ ), lipogenesis (GPDH), and lipolysis (LPL) and (B) inflammation (IL1 $\beta$, IL6, TNF $\alpha$, MCP1, ChemR23, GPR1, and chemerin) in the adipose tissue of 14-month-old WT (black columns, $n=7$ ) and ChemR23 KO (white columns, $n=9$ ) mice was measured by qRT-PCR. The unpaired two-tailed Student's $t$-test was used, ${ }^{*} P<0.05$. (C) Differentiation of the preadipocytes of WT and ChemR 23 KO mice after staining of lipid droplets using Oil Red O (original magnification, $20 \times$ ). (D) Representative quantification (\%) of the differentiated cells in WT and ChemR23 KO mice. Black columns, WT mice; white columns, ChemR23 KO mice.

mature-onset obesity phenotype when compared with their WT littermates. This obesity is associated with a significant increase in body fat content. We did not observe any change in food intake or a decrease in energy expenditure that might explain the progressive increase in body weight. However, given the slow development of this obesity, the difference in energy balance might remain below the level of detection, particularly for food intake, which is difficult to assess precisely. This phenotype is not in agreement with the recently published data reporting lower body mass, body fat percentage, and food intake in ChemR23 KO mice (Ernst et al. 2012). Differences in regimen might explain these differences. Indeed, the standard low-fat diets used in both the studies differ $(3.5 \% \mathrm{kcal}$ from fat in the present study vs $10 \%$ in the study of Ernst et al. (2012)). In the present study, we also observed an influence of sex, as obesity did not reach significance in female mice, and therefore data were collected only for male mice. Ernst et al. (2012) did not report the sex of animals used or differences according to sex.

Histological analyses of adipose tissue indicated hypertrophy of adipocytes in ChemR23 KO mice. There was also overexpression of the lipogenic enzyme GPD1, a major actor in lipid biogenesis acting at the level of glycerol synthesis, suggesting a role for ChemR23 in the negative control of lipogenesis. Previous in vitro studies have shown that recombinant chemerin could enhance lipolysis in adipocytes (Roh et al. 2007), while knockdown of the gene encoding chemerin has been reported to inhibit lipolysis (Goralski et al. 2007). These in vitro data are consistent with the in vivo results of the present study and further support the hypothesis that chemerin has an important role in the negative control of the lipolytic function of adipocytes.

Recently, a chemerin-dependent exacerbation of glucose intolerance has been observed in mouse models of obesity and diabetes (Ernst et al. 2010), while, conversely, glucose intolerance has also been reported in ChemR23 KO mice (Ernst et al. 2012). In the present study, the KO of ChemR23 led to increased body weight but not to basal hyperglycemia or deficient insulin secretion, even in the context of glucose or insulin tolerance tests. In 14-monthold mice, no difference in basal insulin levels was observed, despite the difference in fat mass between WT and ChemR23 KO mice. Ernst et al. (2012) reported a reduction in glucose-induced insulin secretion in vivo, but no effect of ChemR23 knockdown on insulin release in murine MIN6 pancreatic $\beta$-cells, suggesting that the involvment of the chemerin/ChemR23 system might be indirect.

Published by Bioscientifica Ltd 
Mice fed a HFD have been characterized as a model of obesity and associated type 2 diabetes (Winzell \& Ahren 2004). Such a regimen indeed leads to increased body weight gain and progressive alteration of glucose tolerance. On being fed a HFD, no difference in body weight and cholesterol levels were observed between ChemR23 KO and control mice, indicating that the chemerin/ChemR23 system has more effect under normocaloric conditions. HFD promoted the upregulation of chemerin expression in WAT, in accordance with the findings reported by Bauer et al. (2011), and ChemR23 expression was also upregulated. The upregulation of chemerin expression in adipose tissue was not associated with higher chemerin levels in blood, suggesting that WAT might not represent the main source of circulating chemerin, even in mice with increased adipose tissue mass. Despite the increase in fat mass, we did not observe an increase in local or circulating levels of cytokines or their transcripts.

Previous in vitro studies have established a correlation between adipocyte differentiation stage and chemerin expression levels (Bozaoglu et al. 2007, Goralski et al. 2007, Roh et al. 2007, Takahashi et al. 2008) and indicated a major role for the chemerin/ChemR23 axis in the control of the differentiation of mesenchymal stem cells into mature adipocytes (Muruganandan et al. 2010, 2011). The differentiation of preadipocytes into adipocytes requires the suppression of the signaling of the preadipocyte marker PREF1 (Sul et al. 2000, Sul 2009). In the present study, the adipose tissue of ChemR23 KO mice appeared to be mature and functional. Similar expression levels of PREF1, lipin 1, and the adipocyte differentiation marker PPAR $\gamma$ were observed in both the genotypes. Fat tissue mass was observed to increase in (male) KO mice, with no apparent change in the number of cells, but with an increase in lipid accumulation in each cell. Such observations do not appear to support the conclusions of previous in vitro studies and the major role of chemerin and ChemR23 in adipocyte differentiation. Moreover, the results of our in vitro studies indicate a similar differentiation of preadipocytes from the adipose tissue of WT and ChemR23 KO mice (Fig. 5C and D). To draw conclusions regarding the real role of the chemerin/ ChemR23 system in adipocyte differentiation, more investigations by independent groups should be carried out in the future using primary cultures of mesenchymal stem cells or preadipocytes obtained from WT and ChemR23 KO mice.

Studies in humans have demonstrated a strong correlation between circulating chemerin levels and markers of inflammation and the metabolic syndrome (Lehrke et al. 2009, Spiroglou et al. 2010, Weigert et al. 2010, Dong et al. 2011, Gao et al. 2011, Ernst et al. 2012). Yet, the circulating levels of TNF $\alpha$ and expression of proinflammatory cytokines in adipose tissue were not increased in ChemR23-deficient mice, even after they had been fed a HFD for 20 weeks. In their recent study, Ernst et al. (2012) have suggested that ChemR23 could be involved in the recruitment of leukocytes to the liver and WAT, as HFD-fed ChemR23 KO mice displayed lower levels of TNF $\alpha$ in the liver and lower levels of TNF $\alpha$ and IL6 in WAT when compared with WT control mice. No such finding was observed in our study. Differences between the two studies include the diets fed to the mice. We chose to use a moderately HFD (46\% lipid-derived calories), because we foresaw that ChemR23 KO mice might display an increased sensitivity to such a diet, while Ernst et al. used a $60 \%$ lipid-derived diet. Also, as has been stated above, it is unclear whether male or female mice (or both) were used in the latter study, and we observed sex-specific differences in terms of body weight gain. Finally, Ernst et al. did not describe the nature of the control group (littermates or independent animals of the C57BL/6 genetic background), and this may significantly affect the results. Throughout our study, we used as controls age-matched WT mice derived from the same breeding colony as the KO mice. Finally, the sanitary status of the animals might contribute to differences, given the role of chemerin in inflammation. The mice used in the present study were maintained in a SPF environment, while the sanitary status of the animals was not specified by Ernst et al.

In summary, through this study, we provide evidence that the chemerin/ChemR23 system is not essential for adipocyte differentiation in vivo, but that it plays a role in the control of body weight and energy metabolism. However, differences in the results of this study and other reports in the literature, as well as between published reports, suggest that this role is complex, associated with other signaling pathways and that the net effect is highly dependent on the precise experimental setting. If we extrapolate our data to correlations previously made for human populations, the overproduction of chemerin in obese patients might constitute an adaptive pathway aimed at correcting metabolic imbalance.

\section{Supplementary data}

This is linked to the online version of the paper at http://dx.doi.org/10.1530/ JOE-13-0106.

Published by Bioscientifica Ltd. 
Declaration of interest

The authors declare that there is no conflict of interest that could be perceived as prejudicing the impartiality of the research reported.

\section{Funding}

This work was supported by the Interuniversity Attraction Poles Programme (P6/14) - Belgian State - Belgian Science Policy, the Actions de Recherche Concertées of the Communauté Française de Belgique, the Fonds de la Recherche Scientifique Médicale of Belgium, the Walloon Region (Programme d'excellence 'CIBLES'), and the Fédération Belge contre le Cancer.

\section{References}

Arch JR, Hislop D, Wang SJ \& Speakman JR 2006 Some mathematical and technical issues in the measurement and interpretation of open-circuit indirect calorimetry in small animals. International Journal of Obesity $\mathbf{3 0}$ 1322-1331. (doi:10.1038/sj.ijo.0803280)

Bauer S, Wanninger J, Schmidhofer S, Weigert J, Neumeier M, Dorn C, Hellerbrand C, Zimara N, Schaffler A, Aslanidis C et al. 2011 Sterol regulatory element-binding protein 2 (SREBP2) activation after excess triglyceride storage induces chemerin in hypertrophic adipocytes. Endocrinology 152 26-35. (doi:10.1210/en.2010-1157)

Bondue B, Wittamer V \& Parmentier M 2011 Chemerin and its receptors in leukocyte trafficking, inflammation and metabolism. Cytokine \& Growth Factor Reviews 22 331-338. (doi:10.1016/j.cytogfr.2011.11.004)

Bozaoglu K, Bolton K, McMillan J, Zimmet P, Jowett J, Collier G, Walder K \& Segal D 2007 Chemerin is a novel adipokine associated with obesity and metabolic syndrome. Endocrinology 148 4687-4694. (doi:10.1210/ en.2007-0175)

Bozaoglu K, Segal D, Shields KA, Cummings N, Curran JE, Comuzzie AG, Mahaney MC, Rainwater DL, VandeBerg JL, MacCluer JW et al. 2009 Chemerin is associated with metabolic syndrome phenotypes in a Mexican-American population. Journal of Clinical Endocrinology and Metabolism 94 3085-3088. (doi:10.1210/jc.2008-1833)

Dong B, Ji W \& Zhang Y 2011 Elevated serum chemerin levels are associated with the presence of coronary artery disease in patients with metabolic syndrome. Internal Medicine 50 1093-1097. (doi:10.2169/ internalmedicine.50.5025)

Ernst MC \& Sinal CJ 2010 Chemerin: at the crossroads of inflammation and obesity. Trends in Endocrinology and Metabolism 21 660-667. (doi:10.1016/j.tem.2010.08.001)

Ernst MC, Issa M, Goralski KB \& Sinal CJ 2010 Chemerin exacerbates glucose intolerance in mouse models of obesity and diabetes. Endocrinology 151 1998-2007. (doi:10.1210/en.2009-1098)

Ernst MC, Haidl ID, Zuniga LA, Dranse HJ, Rourke JL, Zabel BA, Butcher EC $\&$ Sinal CJ 2012 Disruption of the chemokine-like receptor-1 (CMKRL1) is associated with reduced adiposity and glucose intolerance. Endocrinology 153 672-682. (doi:10.1210/en.2011-1490)

Galic S, Oakhill JS \& Steinberg GR 2010 Adipose tissue as an endocrine organ. Molecular and Cellular Endocrinology 316 129-139. (doi:10.1016/ j.mce.2009.08.018)

Gao X, Mi S, Zhang F, Gong F, Lai Y, Gao F, Zhang X, Wang L \& Tao H 2011 Association of chemerin mRNA expression in human epicardial adipose tissue with coronary atherosclerosis. Cardiovascular Diabetology 1087. (doi:10.1186/1475-2840-10-87)

Goralski KB, McCarthy TC, Hanniman EA, Zabel BA, Butcher EC, Parlee SD, Muruganandan S \& Sinal CJ 2007 Chemerin, a novel adipokine that regulates adipogenesis and adipocyte metabolism. Journal of Biological Chemistry 282 28175-28188. (doi:10.1074/jbc.M700793200)

http://joe.endocrinology-journals.org DOI: 10.1530/JOE-13-0106
(C) 2013 Society for Endocrinology Printed in Great Britain
Hu E, Liang P \& Spiegelman BM 1996 AdipoQ is a novel adipose-specific gene dysregulated in obesity. Journal of Biological Chemistry 271 10697-10703. (doi:10.1074/jbc.271.18.10697)

Karlsson EA \& Beck MA 2010 The burden of obesity on infectious disease. Experimental Biology and Medicine 235 1412-1424. (doi:10.1258/ ebm.2010.010227)

Lehrke M, Becker A, Greif M, Stark R, Laubender RP, von Ziegler F, Lebherz C, Tittus J, Reiser M, Becker C et al. 2009 Chemerin is associated with markers of inflammation and components of the metabolic syndrome but does not predict coronary atherosclerosis. European Journal of Endocrinology 161 339-344. (doi:10.1530/EJE-09-0380)

Luangsay S, Wittamer V, Bondue B, De Henau O, Rouger L, Brait M, Franssen JD, de Nadai P, Huaux F \& Parmentier M 2009 Mouse ChemR23 is expressed in dendritic cell subsets and macrophages, and mediates an anti-inflammatory activity of chemerin in a lung disease model. Journal of Immunology 183 6489-6499. (doi:10.4049/ jimmunol.0901037)

MacDougald OA \& Burant CF 2007 The rapidly expanding family of adipokines. Cell Metabolism 6 159-161. (doi:10.1016/j.cmet. 2007.08.010)

Maffei M, Fei H, Lee GH, Dani C, Leroy P, Zhang Y, Proenca R, Negrel R, Ailhaud G \& Friedman JM 1995 Increased expression in adipocytes of $o b$ RNA in mice with lesions of the hypothalamus and with mutations at the $d b$ locus. PNAS 92 6957-6960. (doi:10.1073/ pnas.92.15.6957)

Muruganandan S, Parlee SD, Rourke JL, Ernst MC, Goralski KB \& Sinal CJ 2010 Chemerin, a novel peroxisome proliferator-activated receptor $\gamma$ $(\mathrm{PPAR} \gamma)$ target gene that promotes mesenchymal stem cell adipogenesis. Journal of Biological Chemistry 286 23982-23995. (doi:10.1074/ jbc.M111.220491)

Muruganandan S, Roman AA \& Sinal CJ 2011 Role of chemerin/CMKLR1 signaling in adipogenesis and osteoblastogenesis of bone marrow stem cells. Journal of Bone and Mineral Research 25 222. (doi:10.1359/jbmr. 091106)

Mussig K, Staiger H, Machicao F, Thamer C, Machann J, Schick F, Claussen CD, Stefan N, Fritsche A \& Haring HU 2009 RARRES2 encoding the novel adipokine chemerin, is a genetic determinant of disproportionate regional body fat distribution: a comparative magnetic resonance imaging study. Metabolism 58 519-524. (doi:10.1016/j.metabol.2008.11.011)

Ouchi N, Parker JL, Lugus JJ \& Walsh K 2011 Adipokines in inflammation and metabolic disease. Nature Reviews. Immunology 11 85-97. (doi:10.1038/nri2921)

Parlee SD, Ernst MC, Muruganandan S, Sinal CJ \& Goralski KB 2010 Serum chemerin levels vary with time of day and are modified by obesity and tumor necrosis factor- $\alpha$. Endocrinology 151 2590-2602. (doi:10.1210/en. 2009-0794)

Pi-Sunyer FX 2002 The obesity epidemic: pathophysiology and consequences of obesity. Obesity Research 10(Suppl 2) 97S-104S. (doi:10.1038/oby.2002.202)

Roh SG, Song SH, Choi KC, Katoh K, Wittamer V, Parmentier M \& Sasaki S 2007 Chemerin - a new adipokine that modulates adipogenesis via its own receptor. Biochemical and Biophysical Research Communications 362 1013-1018. (doi:10.1016/j.bbrc.2007.08.104)

Sell H, Divoux A, Poitou C, Basdevant A, Bouillot JL, Bedossa P, Tordjman J, Eckel J \& Clement K 2010 Chemerin correlates with markers for fatty liver in morbidly obese patients and strongly decreases after weight loss induced by bariatric surgery. Journal of Clinical Endocrinology and Metabolism 95 2892-2896. (doi:10.1210/jc.2009-2374)

Spiroglou SG, Kostopoulos CG, Varakis JN \& Papadaki HH 2010 Adipokines in periaortic and epicardial adipose tissue: differential expression and relation to atherosclerosis. Journal of Atherosclerosis and Thrombosis $\mathbf{1 7}$ 115-130. (doi:10.5551/jat.1735)

Sul HS 2009 Minireview: Pref-1: role in adipogenesis and mesenchymal cell fate. Molecular Endocrinology 23 1717-1725. (doi:10.1210/ me.2009-0160) 
Sul HS, Smas C, Mei B \& Zhou L 2000 Function of pref-1 as an inhibitor of adipocyte differentiation. International Journal of Obesity and Related Metabolic Disorders 24(Suppl 4) S15-S19. (doi:10.1038/ sj.ijo.0801494)

Takahashi M, Takahashi Y, Takahashi K, Zolotaryov FN, Hong KS, Kitazawa R, Iida K, Okimura Y, Kaji H, Kitazawa S et al. 2008 Chemerin enhances insulin signaling and potentiates insulin-stimulated glucose uptake in 3T3-L1 adipocytes. FEBS Letters 582 573-578. (doi:10.1016/ j.febslet.2008.01.023)

Weigert J, Neumeier M, Wanninger J, Filarsky M, Bauer S, Wiest R, Farkas S, Scherer MN, Schaffler A, Aslanidis C et al. 2010 Systemic chemerin is related to inflammation rather than obesity in type 2 diabetes. Clinical Endocrinology 72 342. (doi:10.1111/j.1365-2265. 2009.03664.x)
Weir JB 1949 New methods for calculating metabolic rate with special reference to protein metabolism. Journal of Physiology 109 1-9.

Winzell MS \& Ahren B 2004 The high-fat diet-fed mouse: a model for studying mechanisms and treatment of impaired glucose tolerance and type 2 diabetes. Diabetes 53(Suppl 3) S215-S219. (doi:10.2337/ diabetes.53.suppl_3.S215)

Wittamer V, Franssen JD, Vulcano M, Mirjolet JF, Le Poul E, Migeotte I, Brezillon S, Tyldesley R, Blanpain C, Detheux M et al. 2003 Specific recruitment of antigen-presenting cells by chemerin, a novel processed ligand from human inflammatory fluids. Journal of Experimental Medicine 198 977-985. (doi:10.1084/jem.20030382)

Wozniak SE, Gee LL, Wachtel MS \& Frezza EE 2009 Adipose tissue: the new endocrine organ? A review article Digestive Diseases and Sciences 54 1847-1856. (doi:10.1007/s10620-008-0585-3)

Received in final form 2 September 2013

Accepted 1 October 2013

Accepted Preprint published online 1 October 2013
Published by Bioscientifica Ltd. 